\title{
PERFIL DE LESÕES EM PILOTOS DE PARAPENTE NO BRASIL E SEUS FATORES DE RISCO
}

PROFILE OF INJURIES IN PARAGLIDING PILOTS IN BRAZIL AND THEIR RISKFACTORS

Artigo Original

PERFIL DE LESIONES EN PILOTOS DE PARAPENTEEN BRASIL Y SUS FACTORES DE RIESGO

Jackeline Crivellaro ${ }^{\top}$

(Fisioterapeuta)

Renan Moritz Varnier Rodrigues de Almeida² (Engenheiro)

Rodney Wenke' (Fisioterapeuta)

Eduardo Borba Neves 2,3

(Fisioterapeuta)

\section{Centro Universitário Campos de Andrade, Curitiba, PR, Brasil. \\ 2. Universidade Federal do \\ Rio de Janeiro (UFRJ), Programa de Pós-graduação em Engenharia Biomédica, Rio de Janeiro, \\ RJ, Brasil. \\ 3. Instituto de Pesquisa da Capacitação Física do Exército (IPCFEX), Rio de Janeiro, \\ RJ, Brasil}

\section{Correspondência:}

Eduardo Borba Neves

Av. João Luis Alves s/no Fortaleza de São João - Urca, Rio de Janeiro,

RJ, Brasil. 22291-090.

borbaneves@hotmail.com

\section{RESUMO}

Introdução: O parapente é uma atividade de voo livre que utiliza um planador ultraleve flexível para decolagem, evolução em voo e pouso. Apesar do crescente número de praticantes desse esporte no Brasil, não foram encontrados dados epidemiológicos de lesões na prática de parapente no país. Objetivo: Esta pesquisa teve como objetivo analisar a incidência, tipo, área anatômica acometida, gravidade, e também verificar se há algum padrão nas lesões em pilotos de parapente no Brasil. Métodos: Realizou-se um estudo quantitativo, descritivo, analítico, transversal e retrospectivo por meio de um questionário adaptado do Formulário de Notificação de Acidentes do órgão Alemão LBA - Federal Office of Civil Aeronautics - para obter informações pertinentes aos objetivos da pesquisa. A aplicação do questionário foi realizada on-line, pelo Google Formulários. Resultados: Foram contabilizadas 575 participações válidas, sendo 92,7\% dos participantes do sexo masculino, sendo 30,4\% com idades entre 41 e 50 anos. Entre eles, 68,6\% relataram já ter sofrido uma lesão no esporte; a região anatômica de maior incidência foram os membros inferiores $(44,09 \%)$, sendo o pouso, a fase do voo em que aconteceu a maioria das lesões (68,01\%). Uma regressão logística identificou que "idade" e "tipo de homologação" eram os dois fatores associados à ocorrência de lesão na amostra estudada. Conclusão: Com base nesta pesquisa, é possível elaborar um programa de treinamento funcional para os praticantes desta modalidade, visando um melhor desempenho, medidas de prevenção de lesões associadas ao esporte, bem como propiciar a reabilitação adequada aos pilotos de parapente.

Descritores: atletas; esportes; ferimentos e lesões; fatores de risco; aviação.

\section{ABSTRACT}

Introduction: Paragliding is a free-flight activity that uses a flexible ultralight glider for takeoff, flight and landing. Despite the increasing number of practitioners of this sport in Brazil, no epidemiological data on paragliding injuries were found in the country. Objective: This study aimed to analyze the incidence, type, anatomical area affected, severity, and to verify if there is any pattern in the lesions in paraglider pilots in Brazil. Methods: A Quantitative, descriptive, analytical, cross-sectional, retrospective study was carried out using an adapted questionnaire from the Accident Notification Form of the German agency LBA - Federal Office of Civil Aeronautics to obtain information relevant to the research objectives. The application of the questionnaire was conducted online, through Google Forms Results: There were 575 valid participations, of which 92.7\% were male, and 30.4\% were between 41 and 50 years old. Among them, 68.6\% reported having already had an injury in this sport. The anatomical region with the highest injury incidence was the lower limbs (44.09\%), the landing being the flight phase in which most of the lesions occurred (68.01\%). A logistic regression identified that "age" and "type of homologation" were the two factors associated with the occurrence of injury in the studied sample. Conclusion: Based on this research, it is possible to develop a functional training program for the practitioners of this modality, aiming at a better performance, measures of prevention of injuries associated to the sport, as well as to provide adequate rehabilitation to paragliding pilots.

Keywords: athletes; sports; wounds and injuries; risk factors; aviation.

\section{RESUMEN}

Introducción: El parapente es una actividad de vuelo libre que utiliza un planeador ultraligero flexible para despegue, evolución en vuelo y aterrizaje. A pesar del creciente número de practicantes de este deporte en Brasil, no se encontraron datos epidemiológicos de lesiones en la práctica de parapente en el país. Objetivo: Esta investigación tuvo como objetivo analizar la incidencia, tipo, área anatómica afectada, gravedad, y también comprobar si hay algún patrón en las lesiones en pilotos de parapente en Brasil. Métodos: Se realizó un estudio cuantitativo, descriptivo, analítico, transversal, retrospectivo a través de un cuestionario adaptado del Formulario de Notificación de Accidentes de la agencia alemán LBA - Federal Office of Civil Aeronautics para obtener información pertinente a los objetivos de la investigación. Resultados: La aplicación del cuestionario fue realizada en línea por Google Formularios. Se contabilizaron 575 participaciones válidas, siendo el 92,7\% de los participantes masculinos y el 30,4\% con edades entre 41 y 50 años. Entre ellos, el 68,6\% relató haber sufrido una lesión en el deporte; la región anatómica de mayor incidencia fueron los miembros inferiores (44,09\%) y el aterrizaje, la fase de vuelo en que ocurrió la mayoría de las lesiones $(68,01 \%)$. 
Una regresión logística identificó que la "edad"y "tipo de homologación" fueron los dos factores asociados a la ocurrencia de lesiones en la muestra estudiada. Conclusión: Con base en esta investigación, es posible desarrollar un programa de entrenamiento funcional para los practicantes de esta modalidad, buscando un mejor rendimiento, medidas de prevención de lesiones relacionadas al deporte, así como propiciar la rehabilitación adecuada a los pilotos de parapente.

Descriptores: atletas; deportes; heridas y lesiones; factores de riesgo; aviación.

\section{INTRODUÇÃO}

O parapente é uma atividade de voo livre que utiliza um planador ultraleve flexível para decolagem, evolução em voo e pouso. Considerado um esporte de aventura e de alto risco, o voo de parapente teve inicio no Brasil em 1987 e, desde então, o número de pessoas praticando essa modalidade de voo tem crescido'. Com o crescente número de adeptos ao esporte, observa-se a ocorrência de lesões associadas à modalidade. Apesar dos fatores de risco para essas lesões não serem bem conhecidos, muitas delas ocorrem por imperícia do piloto, seja por tentar executar uma ação que não é capaz de dominar, ou por fazer algo que não deveria ser feito².

A Confederação Brasileira de Voo Livre dispõe de normas de formação de pilotos, nivelamento, requisitos e prerrogativas com o objetivo de minimizar a ocorrência de acidentes. Por meio desses requisitos, os pilotos são enquadrados em níveis, devendo utilizar equipamentos de voo conforme sua habilitação ${ }^{3}$. Além dos níveis de habilitação, existem normas de homologação de instrutores, e o parapente é classificado de acordo com sua aeronavegabilidade, sendo o comportamento do equipamento em voo testado e avaliado. A classificação da vela é realizada de acordo com a nota obtida nesse teste, fornecendo uma orientação ao piloto sobre o parapente $e^{4,5}$.

Como em outros esportes, o parapente apresenta um potencial de risco de lesões, que podem ser predispostas por fatores intrínsecos ou extrínsecos ao equipamento ${ }^{6}$. Para compreender quais são estes possíveis fatores, bem como para elaborar diretrizes de prevenção para pilotos de parapente, há necessidade de conhecer a epidemiologia das lesões ocorridas nesta modalidade do voo livre. No entanto, não foram encontrados estudos epidemiológicos de lesões na prática de parapente no Brasil, impossibilitando o desenvolvimento de estratégias de prevenção. Assim, esta pesquisa tem como objetivo analisar a incidência, tipo, área anatômica acometida, gravidade e padrão nas lesões em pilotos de parapente no Brasil.

\section{MATERIAIS E MÉTODOS}

Esta pesquisa foi aprovada pelo comitê de ética do Centro Universitário Campos de Andrade, sob o número CAAE 53627216.7.0000.5218. Todos os participantes assinaram o Termo de Consentimento Livre e Esclarecido. Realizou-se um estudo quantitativo, do tipo descritivo analítico com corte transversal, retrospectivo por meio de um questionário adaptado do formulário de notificação de acidentes do órgão Alemão LBA - Federal Office of Civil Aeronautics, para obter informações de pilotos de parapente habilitados para voo no Brasil.

Os critérios de inclusão foram: o indivíduo deveria ser habilitado pela Confederação Brasileira de Voo Livre ou pela Associação Brasileira de Parapente. A aplicação do questionário foi realizada via on-line, no período de 26 de fevereiro de 2016 a 20 de maio do mesmo ano. Foi utilizado o Google formulários para responder ao questionário disponibilizado aos praticantes da modalidade. O convite para participação dos pilotos foi por meio da divulgação de associações, federações e clubes da modalidade esportiva, e-mail, bem como nas redes sociais, no Facebook e Instagram, nos perfis e páginas relacionadas a parapente no Brasil.

As seguintes variáveis foram analisadas: Idade; Tempo de voo, Tempo de habilitação à data da lesão; Nível de habilitação; Tipo de homologação; Região da Lesão; Momento da lesão, Tipo de calçado e Atividade física. Os níveis de habilitação são classificados progressivamente como: aluno em instrução; nível 1; nível 2; nível 3; nível 4; nível 5 . O tipo de homologação do equipamento é classificado como " $A$ " (mais seguro, indicado para pilotos em todos os níveis de formação) e " $B$ ", "C", "D" e "CCC", progredindo quanto à exigência de habilidade e experiência do piloto (equipamentos mais dinâmicos, mas com uma resposta mais violenta à turbulência e aos erros de pilotagem) ${ }^{4,5}$.

Inicialmente foi realizado um teste Qui-quadrado considerando a categoria com menor frequência de lesão como valor esperado para as variáveis: Idade; Nível de habilitação; Tempo de habilitação e Atividade física. Para a variável, Tipo de homologação, considerou-se o Perfil Seguro (A - B) como valor esperado. A seguir, foi realizada uma regressão logística na qual Idade foi dicotomizada como " $\leq 40$ anos" vs "> 40 anos"; Nível de habilitação como " $\leq 2$ " vs" $>$ 2"; Tempo de habilitação como " $\leq 5$ anos" vs "> 5 anos"; Tipo de homologação como "A ou B"vs" C, D ou CCC"; Tipo de calçado como "Bota" vs "outros" e Atividade física como "pratica" vs "não pratica". Para essa regressão logística, inicialmente procedeu-se a uma análise univariada $\left(x^{2}\right)$ das associações entre as variáveis dicotomizadas descritas acima e a ocorrência de lesão (dicotomizada como "sim" vs "não") A seguir, as variáveis com associação univariada marginal com a variável desfecho ( $p$-valor $<0,25$ ) foram introduzidas em uma regressão logística, tendo ocorrência de lesão como variável desfecho. A análise foi realizada no programa SPSS v.20.

\section{RESULTADOS}

De acordo com os dados obtidos no "site" da Confederação Brasileira de Voo Livre, até o final de 2015 havia em torno de 4.000 pilotos associados, vinculados a 12 Federações Estaduais cadastradas na Confederação?. O número de indivíduos que responderam à pesquisa foi de 625 , sendo que 50 foram excluídos deste estudo por não cumprirem os critérios de inclusão, resultando em 575 pilotos que responderam ao questionário. As características demográficas principais dos respondentes e as associações univariadas entre as variáveis analisadas e a ocorrência de lesões estão descritas nas Tabelas 1 e 2. Quando a categoria de tempo de habilitação foi dicotomizada em "< 10 anos" e "> 10 anos", o teste Qui-quadrado de Pearson $\left(X^{2}\right)$ indicou diferença estatística significativa $(p=0,030)$ entre a frequência de lesões dos sujeitos que tem mais de 10 anos de habilitação que os demais.

Um total de 372 pilotos $(68,6 \%)$ relatou já terem sofrido lesão no parapente; destes, 31,4\% tiveram mais de uma. Os indivíduos que relataram o maior índice de lesões no esporte foram aqueles de faixas etárias elevadas. Dos 314 diagnósticos referidos, 44,9\% foram de fraturas, 16,24\% luxações, $12,74 \%$ contusões, 10,83\% entorse; o estiramento muscular ou ligamentar foram 7,32\% e outras lesões representam 7,96\%, sendo necessária intervenção cirúrgica em 30,89\% destes casos. 
Tabela 1. Características de praticantes de voo em parapente e de suas lesões, informações obtidas por questionário "online", Brasil, $2016(n=575)$.

\begin{tabular}{|c|c|c|}
\hline Característica & Número & $\%$ \\
\hline \multicolumn{3}{|c|}{ Pilotos } \\
\hline \multicolumn{3}{|l|}{ Nível de habilitação } \\
\hline 1 & 177 & 30,8 \\
\hline 2 & 190 & 33,0 \\
\hline 3 & 162 & 28,2 \\
\hline 4 & 42 & 7,3 \\
\hline 5 & 4 & 0,7 \\
\hline \multicolumn{3}{|c|}{ Tempo de habilitação à data da lesão (anos) } \\
\hline Em instrução & 3 & 0,8 \\
\hline$<0.5$ & 46 & 12,4 \\
\hline $0,5-1$ & 77 & 20,7 \\
\hline $1-2$ & 71 & 19,1 \\
\hline $2-5$ & 93 & 25,0 \\
\hline $5-10$ & 58 & 15,6 \\
\hline$>10$ & 24 & 6,4 \\
\hline \multicolumn{3}{|c|}{ Lesões } \\
\hline \multicolumn{3}{|l|}{ Região da lesão } \\
\hline Cabeça & 3 & 0,8 \\
\hline Coluna vertebral & 102 & 27,4 \\
\hline Membros inferiores & 164 & 44,1 \\
\hline Membros superiores & 59 & 15,9 \\
\hline$>1$ Região & 44 & 11,8 \\
\hline \multicolumn{3}{|l|}{ Momento da lesão } \\
\hline Decolagem & 82 & 22,0 \\
\hline Em voo & 20 & 5,4 \\
\hline Pouso & 253 & 68,0 \\
\hline$>24 \mathrm{hs}$ voo & 17 & 4,6 \\
\hline
\end{tabular}

Tabela 2. Associação entre variáveis selecionadas e ocorrência de lesões em pilotos de parapente, Brasil, 2016 ( $n=575)$. Valores em itálico esperados para calculo da estatística Qui-quadrado.

\begin{tabular}{|c|c|c|c|c|c|}
\hline \multirow{2}{*}{ Variável } & \multicolumn{3}{|c|}{ Lesões } & \multirow{2}{*}{$\begin{array}{l}\text { \% com } \\
\text { lesão }\end{array}$} & \multirow{2}{*}{$\mathrm{x}^{2} / \mathrm{p}$-valor } \\
\hline & 0 & 1 & $>1$ & & \\
\hline \multicolumn{6}{|l|}{ Tipo de homologação } \\
\hline Sem homologação & 2 & 3 & 2 & 71,43 & \multirow{3}{*}{$2,96 / 0,228$} \\
\hline Perfil menos seguro (C-D - CCC) & 65 & 137 & 15 & 70,05 & \\
\hline Perfil seguro $(A-B)$ & 136 & 190 & 25 & 61,25 & \\
\hline \multicolumn{6}{|l|}{ Tipo de selete } \\
\hline Carenada com airbag & 6 & 16 & 2 & 75,00 & \multirow{6}{*}{$5,02 / 0,414$} \\
\hline Carenada sem airbag & 64 & 104 & 13 & 64,64 & \\
\hline Com airbag & 59 & 97 & 10 & 64,46 & \\
\hline Montanha com airbag & 8 & 12 & 3 & 65,22 & \\
\hline Montanha sem airbag & 3 & 4 & 5 & 75,00 & \\
\hline Sem airbag & 63 & 97 & 9 & 62,72 & \\
\hline \multicolumn{6}{|l|}{ Idade (anos) } \\
\hline $18-25$ & 12 & 12 & 3 & 55,56 & \multirow{6}{*}{$16,55 / 0,005$} \\
\hline $26-30$ & 25 & 27 & 2 & 53,70 & \\
\hline $31-35$ & 49 & 53 & 9 & 55,86 & \\
\hline $36-40$ & 42 & 65 & 5 & 62,50 & \\
\hline $41-50$ & 53 & 107 & 15 & 69,71 & \\
\hline$>51$ & 22 & 66 & 8 & 77,08 & \\
\hline \multicolumn{6}{|l|}{ Tempo de voo (anos) } \\
\hline $0,5-1$ & 13 & 17 & 3 & 60,61 & \multirow{5}{*}{$6,83 / 0,145$} \\
\hline $1-2$ & 34 & 52 & 4 & 62,22 & \\
\hline $2-5$ & 79 & 104 & 10 & 59,07 & \\
\hline $5-10$ & 50 & 73 & 10 & 62,41 & \\
\hline$>10$ & 27 & 84 & 15 & 78,57 & \\
\hline \multicolumn{6}{|l|}{ Atividade física (x semana) } \\
\hline 1 & 28 & 30 & 4 & 54,84 & \multirow{5}{*}{$12,10 / 0,017$} \\
\hline 2 & 39 & 83 & 13 & 71,11 & \\
\hline 3 & 52 & 70 & 4 & 58,73 & \\
\hline 4 & 33 & 77 & 12 & 72,95 & \\
\hline Não prática & 49 & 72 & 9 & 62,31 & \\
\hline
\end{tabular}

A Tabela 3 apresenta os resultados da regressão logística para a variável desfecho "ocorrência de lesão". Apenas as variáveis Idade ( $p<0,0001)$ e Tipo de homologação $(p=0,049)$ puderam ser retidas na regressão ( $x^{2}$ geral para o modelo $\left.p<0,001\right)$.

Tabela 3. Regressão logística para fatores de risco relacionados à lesão por voo em parapente, informações obtidas por questionário "online", Brasil, 2016 ( $n=575)$. Variável desfecho "ocorrência de lesão". Categorias de referência: Idade: > 40 anos; Tipo de Homologação: "perfil seguro" (vide texto). $x^{2}$ geral para os coeficientes do modelo $\mathrm{p}<0,001$.

\begin{tabular}{c|c|c|c|c|c|c}
\hline Variáveis & B & S.E. & df & p-valor & OR & IC 95\% \\
\hline Idade & $-0,68$ & 0,18 & 1 & 0,000 & 0,51 & $0,34-0,73$ \\
\hline Tipo de homologação & 0,37 & 0,19 & 1 & 0,049 & 0,05 & $1,45-1,00$ \\
\hline
\end{tabular}

\section{DISCUSSÃO}

As lesões, definidas como dano físico que ocorre após uma súbita ou breve exposição do corpo humano a níveis de energia intoleráveis ou à falta de elementos vitais, são um grave problema de saúde pública em todo o mundo. As atividades esportivas são consideradas um dos eventos mais comuns para causar esse tipo de dano ${ }^{8}$. Por outro lado, o parapente é um esporte de alto risco, com uma grande incidência de lesões graves e fatais, apesar da taxa de ocorrências, em 2003, ser menor que outros esportes aéreos e em comparação ao motociclismo 9 .

No entanto, não existem, até o momento, informações epidemiológicas sobre essas lesões. A Associação Brasileira de Parapente organizou uma Comissão de Estudos de Acidentes (CPEA) em 2010, com os objetivos de sistematizar a análise dos acidentes na prática do voo de parapente, identificar os fatores que causaram os acidentes, e também desenvolver modos de prevenção desses acidentes em todo território nacional. A associação disponibiliza em seu site um formulário para que os pilotos associados reportem os acidentes ocorridos, porém ainda não foram divulgados dados obtidos por esta comissão ${ }^{10}$

A prática de atividade física é um componente utilizado para avaliação das condições de saúde, por sua influência na qualidade de vida em suas fases ${ }^{11,12}$. Knuth et al. ${ }^{13}$ em análise da Pesquisa Nacional por Amostra de Domicílios (PNAD 2008), evidenciou que indivíduos ativos no lazer representam 10,5\% da população. Para essa classificação, os indivíduos deveriam praticar atividade física leve ou moderada por no mínimo trinta minutos, em cinco dias ou mais na semana, ou praticar atividade vigorosa com pelo menos vinte minutos, em no mínimo três dias na semana. Na presente pesquisa a grande maioria dos participantes $(72,3 \%)$ realizava outra atividade física, além do parapente, e, desses $70,2 \%$ praticam atividades que se enquadram como vigorosas três ou mais vezes na semana. Isso pode indicar que a causa das lesões esteja relacionada a acidentes no esporte, já que 68,6\% dos indivíduos tiveram dano físico em parapente, apesar de serem ativos no lazer.

O estudo identificou que os indivíduos com faixa etária e tempo de habilitação em parapente mais elevado foram o grupo com o maior índice de lesões. Isso demonstra, logicamente, que pilotos com maior tempo de exposição na prática de parapente têm uma probabilidade maior de sofrer lesões relacionadas ao esporte. No entanto, a análise descritiva identificou que após dez anos de habilitação a frequência das lesões diminui. Fica evidente, ainda, que os dois primeiros anos de formação são os mais críticos na ocorrência de lesões, já que foi durante este período que aconteceram 52,16\% das lesões mencionadas na pesquisa. Esse dado também foi apontado por Schulze et al. ${ }^{14}$, a partir da análise de 409 acidentes de parapente: apenas três pilotos tiveram lesões enquanto estavam em instrução. Portanto, o número de ocorrências registradas nos dois primeiros anos pode ser um indicativo de que a formação destes indivíduos foi precoce, sem o nível adequado de conhecimento no esporte. 
Kruger-Frank et al. ${ }^{15}$ analisou 218 acidentes com parapente, nos quais a área anatômica com maior número de lesões foram nos membros inferiores (41,3\%), seguidos da coluna vertebral (34,9\%), mesmos locais onde predominaram as lesões investigadas na presente pesquisa. Outros autores $^{16}$ investigaram 64 acidentes de parapente entre agosto de 2004 e setembro de 2011, por meio de revisão de prontuários médicos de três hospitais na região de Fethiye, Turkia, e observaram que as regiões mais afetadas durante os acidentes também foram a coluna lombar (19,44\%) e a articulação do tornozelo (17,59\%).

Para Zeller et al. ${ }^{17}$ a maioria dos acidentes que acometem pilotos de parapente acontecem no momento do pouso, dado que condiz com os resultados deste estudo. Analisando outros estudos sobre lesões em pilotos de parapente ${ }^{18,19}$, pode-se identificar uma preocupação especial dos pesquisadores com as lesões na coluna vertebral, e ainda, que as lesões nessa região acontecem predominantemente durante a aterragem, já que o pouso é o momento no qual se impõe uma grande força de impacto nos membros inferiores e na coluna vertebral, sendo o risco de lesões agravado por irregularidades, obstáculos ou erros na técnica de pouso.
Gosteli et al. ${ }^{20}$ investigaram a epidemiologia das lesões em esportes radicais, com base numa analise retrospectiva das missões de resgate realizadas em Lausanne, Suíça, de 1 de Janeiro de 1998 a 31 Dezembro de 2008, e observou que entre os esportes aéreos (asa delta, paraquedismo e parapente), o parapente foi responsável por $80 \%$ das lesões estudadas. Tais achados reforçam a importância de se identificar os fatores relacionados a essas lesões e a busca de ações práticas para minimizar esses números.

\section{CONCLUSÃO}

Este estudo visou a estudar os fatores, compreendendo as características dos sujeitos e as condições na prática do esporte, que podem ter determinado a ocorrência das lesões em praticantes de parapente no país. Foi possível obter um perfil dos pilotos e destas ocorrências, porém a notificação efetiva dos acidentes de parapente em um sistema nacional é uma ferramenta importante para uma análise mais específica e fiel destas informações.

Todos os autores declararam não haver qualquer potencial conflito de interesses referente a este artigo.

CONTRIBUIÇÕES DOS AUTORES: Cada autor contribuiu individual e significativamente para o desenvolvimento do manuscrito. JC (0000-0002-7593-6394)* e EBN (0000-0003-4507-6562)* foram os principais contribuintes na concepção do estudo e na redação do manuscrito. EBN e RMVRA (0000-0003-0158-7432)* trabalharam na análise estatística e interpretação dos dados. JC, EBN, RMVRA e RW (0000-0001-8862-0324)* realizaram a pesquisa bibliográfica, a revisão do manuscrito e contribuíram com o conceito intelectual do estudo. *ORCID (Open Researcher and Contributor ID).

\section{REFERÊNCIAS}

1. Arqué M. Parapente: iniciação. São Paulo: Perfil Brasil; 2013.

2. Fedération Aéronautique Internationale. ParaPro. Recommended international paragliding standards of safety and training. Switzerland; 2008. Disponivel em: http://www.lzs-zveza.si/panoge/ jadralno-padalstvo-in-zmajarstvo/ippi/parapro/

3. Confederação Brasileira de Voo Livre. Norma regulamentar da Associação Brasileira de Voo Livre ABVL/ Confederação Brasileira de Voo Livre CBV; 2015. Disponível em: http://www.abvl.net/documentos-oficiais. [acesso em 2015 out 10].

4. Peter Wild. Airworthiness requirements for hang gliders and paragliders - LTF-2009. Deutscher Hängegleiterverband e.V. (DHV) Technical department; 2009. Disponível em: http://www.tinyurl.com/ hswx78f. [acesso em 2015 set 27].

5. Federation Aeronautique Internationale. CIVL Competition Class (CCC). Paragliders permitted. In: FAI category 1 Cross-Country events; 2016. Disponivel em: http://www.fai.org/civl-our-sport/competitionclass- paragliders. [acesso em 2015 set 27].

6. Gould JA. Fisioterapia na ortopodia e na medicina do esporte. São Paulo: Manole; 1993.

7. Confederação Brasileira de Voo Livre. Notícias e Imprensa. Disponível em: http://www.abvl. net/que-venham- mais-40- anos-de- voo-livre- obrigado-brasil- \%e2\%80\%8f. [acesso em 2016 abr 24].

8. Organização Mundial de Saúde. Manual de vigilância das lesões. São Paulo: Secretaria de Estado da Saúde de São Paulo; 2004 Imprensa. Disponível em: ftp://ftp.cve.saude.sp.gov.br/doc_tec/outros/ man_lesoes.pdf. [acesso em 2016 abr 24].

9. Bohnsack M, Schröter E. Injury patterns and typical stress situations in paragliding. Orthopade; 2005:34(5);411-8.
10. Associação Brasileira de Parapente. Comissão de Prevenção e Estudo de Acidentes. Disponível em: www.abp.esp.br/cpea. [acessado em 2015 out 10].

11. Souza MdC, Pelegrini A, Silva MCd, Machado Z, Guimarães ACdA. Fatores associados à prática de atividade física em homens de meia-idade. Rev Bras Med Esporte. 2016;22(2):102-7.

12. Organização Mundial da Saúde. Global recommendations on physical activity for health. Geneva: OMS; 2010. Disponível em: http://www.who.int/dietphysicalactivity/publications/9789241599979/ en/. [acesso em 2016 abr 24].

13. Knuth AG, Malta DC, Dumith SC, Pereira CA, Moraes Neto OL, Temporão JC, et al. Prática de atividade física e sedentarismo em brasileiros: Resultados da Pesquisa Nacional por Amostra de Domicílios (PNAD) - 2008. Ciênc Saúde Coletiva. 2011;16(9):3697-705.

14. Schulze W, Richter J, Schulze B, Esenwein SA, Buttner-Janz K. Injury Prophylaxis in paragliding. Br J Sports Med. 2002;36(5):365-9.

15. Kruger-Frank M, Siebert CH, Pforringer W. Paragliding Injuries. Br J Sports Med. 1991;25(2):98-101.

16. Canbek U, Imerci A, Akgun U, Yesil M, Aydin A, Balci Y. Characteristics of injuries caused by paragliding accidents: a cross-sectional study. World J Emerg Med. 2015;6(3):221-4.

17. Zeller T, Billing A, Lob G. Injuries in paragliding. Int Orthop. 1992;16:255-9.

18. Rekand T, Schaanning E, Varga V, Schattel U, Gronning M. Spinal cord injuries among paragliders in Norway. Spinal Cord. 2008;46(6):412-6.

19. Hasler RM, Hüttner HE, Keel MJ, Durrer B, Zimmermann H, Exadaktylos AK, et al. Spinal and pelvic injuries in airborne sports: a retrospective analysis from a major Swiss trauma centre. Injury. 2012;43(4):440-5.

20. Gosteli G, Yersin B, Mabire C, Pasquier M, Albrecht R, Carron PN. Retrospective analysis of 616 air-rescue trauma cases related to the practice of extreme sports. Injury. 2016;47:1414-20. 